\title{
Tratamiento de las Infecciones Urinarias con una Monodosis Diaria de Gentamicina (Refobacin)
}

\author{
Dres.: Joaquín Luna $\mathrm{R}^{*}$, Joaquín Luna $\mathrm{U}^{* *}$
}

INTRODUCCION

Es bien conocida la alta frecuencia de infecciones del tracto urinario en la mujer. También es conocido que la mayoría de estas infecciones son causadas por gérmenes gram-negativos que responden a una variada gama de antibióticos; pero también se sabe que muchos de estos antibióticos han perdido campo frente a estos gérmenes debido a un mal uso terapéutico.

Desde hace mucho tiempo se han utilizado con mucho éxito los antibióticos del grupo de los amino-glucósidos en el tratamiento de estas infecciones con base en los altos niveles que se logran del antibiótico en el tejido renal, las vías urinarias y orina (1); además la sensibilidad de los agentes patógenos frente a este grupo de antibióticos permanece casi inmodificada a pesar de la prolongada utilización clínica. Se destaca el uso de la gentamicina por los buenos resultados obtenidos en los tratamientos y la escasa aparición de resistencias en razón de su triple molécula antibiótica por lo cual es en la actualidad un antibiótico confiable (1).

Frecuentemente se ha utilizado como forma de tratamiento la administración de dosis cada 8 ó 12 horas (2). Desde hace algunos años, diferentes autores han propuesto la administración de una monodosis diaria equivalente a las dos terceras partes de la dosis total diaria de la suministrada cada 8 horas $(2,3)$. Esto no es nuevo, puesto que desde 1963 se observó que tras una dosis única de gentamicina al día, los niveles séricos óptimos permanecían por

Residente III Ginecología y Obstetricia Hospital de La Samaritana.

Coordinador Depto. de Ginecología y Obstetricia Hospital de La Samaritana.
12 horas y los niveles terapéuticos en la orina se demostraron hasta por 24 horas (4).

Basados en esto se propuso desde 1973 en varias publicaciones $(5,6)$ la administración de una monodosis diaria de gentamicina para el tratamiento de las infecciones del tracto urinario demostrándose que el efecto terapéutico era comparable con la administración de tres dosis diarias $(2,3)$.

Como un hecho adicional se facilita a estas pacientes el cumplir el tratamiento ambulatorio y presenta un riesgo disminuido de oto y nefro toxicidad como efectos secundarios (1 - 3).

Se propuso realizar un trabajo utilizando gentamicina (Refobacin) en una dosis única diaria de 2 $\mathrm{mg} / \mathrm{kg}$ de peso en el tratamiento de infecciones urinarias en mujeres y comprobar la eficacia de este esquema terapéutico y evaluar la sensibilidad del urocultivo frente a la respuesta clínica de la paciente.

\section{MATERIALES Y METODOS}

Se realizó un estudio prospectivo no comparativo en 21 pacientes ambulatorias de la consulta externa de ginecología. Se incluyeron al estudio mujeres voluntarias que cumplieran con los criterios de selección, es decir, tener una edad entre 20 y 70 años y que presentaran un cuadro clínico de infección urinaria caracterizado por fiebre, disuria, poliaquiuria, nicturia y tenesmo vesical, excluyéndose aquellas pacientes con antecedentes de nefropatía, insuficiencia renal, hipertensión reno-vascular o enfermedades severas que incluyeran terapias concomitantes de uso prolongado.

También se excluyeron las pacientes que hubieran tomado previamente antibióticos o tuvieran hipersensibilidad conocida a la gentamicina. 
A todas las pacientes se les elaboró una historia clínica que incluía los datos de identificación, evaluación de síntomas y exámenes paraclínicos al ingreso y en los controles.

Los síntomas se evaluaron al ingreso, cuarto y séptimo días de tratamiento donde se tomaron los exámenes paraclínicos (BUN, parcial de orina al ingreso - día cero- y urocultivo al ingreso y en los controles). Los síntomas se evaluaron de acuerdo con la severidad en la escala de cero $(0=$ ausente) a tres $(3=$ severo $)$. El urocultivo se consideró positivo cuando creció un solo germen y el recuento de colonias fue igual o superior a 100.000.

Las pacientes recibieron un tratamiento con gentamicina durante 7 días a una dosis única diaria de $2 \mathrm{mg} / \mathrm{kg}$ de peso día.

La respuesta terapéutica se evaluó globalmente como excelente, cuando el urocultivo fue negativo y la sintomáticos desapareció. Bueno, cuando el cultivo fue negativo y los síntomas habían disminuido a leves. Regular, cuando la variación de los síntomas fue moderada o sin urocultivo negativo. Mala, cuando hubo modificaciones en la sintomáticos o en el urocultivo.

\section{METODOS ESTADISTICOS}

Los resultados se presentan en tablas y gráficas, Se utilizaron como pruebas estadísticas análisis de varianza y la "CHI CUADRADO" para proporciones y la prueba de FISHER. Se consideró significativo $\mathrm{p}<0.05$.

\section{RESULTADOS}

Ingresaron al estudio 21 pacientes (mujeres) que cumplieron con los requisitos de admisión, de las cuales 18 fueron evaluables a los 17 días, las otras 3 pacientes no fueron evaluables porque no regresaron a los controles.

En la Tabla 1 se observan los datos generales sobre edad y peso y los exámenes paraclínicos de BUN y creatinina, los cuales estuvieron dentro de los límites normales.

Los síntomas evaluados fueron fiebre, disuria, poliaquiuria, nicturia y tenesmo. Todos los pacientes tuvieron al menos dos síntomas.

La fiebre estuvo presente en forma leve en tres casos, la cual desapareció en el control del cuarto
TABLA 1

DATOS GENERALES

\begin{tabular}{|lrr|}
\hline & \multicolumn{1}{c|}{ X } & SD \\
\hline Edad (a los) & 51.5 & 11.3 \\
Peso (Kg) & 58.8 & 7.5 \\
& & \\
Laboratorio & 14.8 & 2.5 \\
Bun (mg \%) & 0.8 & 0.2 \\
Creatinina (mg \%) & & \\
\hline
\end{tabular}

día. La disuria estuvo presente en 16 casos entre leve o severa y en la evaluación del día 17 sólo un caso mantuvo este síntoma como moderado. La poliaquiuria estuvo presente en 15 casos y al final de la observación 4 casos la presentaron como leve. La nicturia la presentaron 14 pacientes, lo mismo que la poliaquiuria, al final del estudio la mantuvieron 4 pacientes. El tenesmo se presentó en 7 casos los cuales al control del cuarto día había desaparecido (Tabla 2, Figura 1).

TABLA 2

FRECUENCIA DE PACIENTES SEGUN SEVERIDAD DE SINTOMAS DURANTE EL TIEMPO DE OBSERVACION

\begin{tabular}{|c|c|c|c|c|c|c|c|c|c|}
\hline \multirow[b]{2}{*}{ Sintomas } & \multirow{2}{*}{$\begin{array}{c}\text { Severidac } \\
\text { Días }\end{array}$} & \multicolumn{2}{|c|}{ Ausente } & \multicolumn{2}{|c|}{ Leve } & \multicolumn{2}{|c|}{ Moderado } & \multicolumn{2}{|c|}{ Severo } \\
\hline & & N & $\%$ & $\mathbf{N}$ & $\%$ & $\mathbf{N}$ & $\%$ & $\mathbf{N}$ & $\%$ \\
\hline \multirow[t]{4}{*}{ Fiebre } & 0 & 15 & .83 .3 & 3 & 16.7 & 0 & 0.0 & 0 & 0.0 \\
\hline & 4 & 18 & 100.0 & 0 & 0.0 & 0 & 0.0 & 0 & 0.0 \\
\hline & 7 & 18 & 100.0 & 0 & 0.0 & 0 & 0.0 & 0 & 0.0 \\
\hline & 17 & 18 & 100.0 & 0 & 0.0 & 0 & 0.0 & 0 & 0.0 \\
\hline \multirow[t]{4}{*}{ Disuria } & 0 & 2 & 11.1 & 11 & 61.1 & 2 & 11.1 & 3 & 16.7 \\
\hline & 4 & 8 & 44.4 & 10 & 55.6 & 0 & 0.0 & 0 & 0.0 \\
\hline & 7 & 13 & 72.2 & 4 & 22.2 & 1 & 5.6 & 0 & 0.0 \\
\hline & 17 & 17 & 94.4 & 0 & 0.0 & 1 & 5.6 & 0 & 0.0 \\
\hline \multirow[t]{4}{*}{ Poliaquiuria } & 0 & 3 & 16.7 & 3 & 16.7 & 10 & 55.6 & 2 & 11.1 \\
\hline & 4 & 8 & 44.4 & 6 & 33.3 & 4 & 22.2 & 0 & 0.0 \\
\hline & 7 & 11 & 61.1 & 6 & 33.3 & 1 & 5.6 & 0 & 0.0 \\
\hline & 17 & 14 & 77.8 & 4 & 22.2 & 0 & 0.0 & 0 & 0.0 \\
\hline \multirow[t]{4}{*}{ Nicturia } & 0 & 4 & 22.2 & 9 & 50.0 & 4 & 22.2 & 1 & 5.6 \\
\hline & 4 & 12 & 66.7 & 4 & 22.2 & 2 & 11.1 & 0 & 0.0 \\
\hline & 7 & 13 & 72.2 & 4 & 22.2 & 1 & 5.6 & 0 & 0.0 \\
\hline & 17 & 14 & 77.8 & 4 & 22.2 & 0 & 0.0 & 0 & 0.0 \\
\hline \multirow[t]{4}{*}{ Tenesmo } & 0 & 11 & 61.1 & 6 & 33.3 & 1 & 5.6 & 0 & 0.0 \\
\hline & 4 & 18 & 100.0 & 0 & 0.0 & 0 & 0.0 & 0 & 0.0 \\
\hline & 7 & 18 & 100.0 & 0 & 0.0 & 0 & 0.0 & 0 & 0.0 \\
\hline & 17 & 18 & 100.0 & 0 & 0.0 & 0 & 0.0 & 0 & 0.0 \\
\hline
\end{tabular}


FIGURA 1

TOTAL DE PUNTOS PROMEDIO

DEL TOTAL DE SINTOMAS

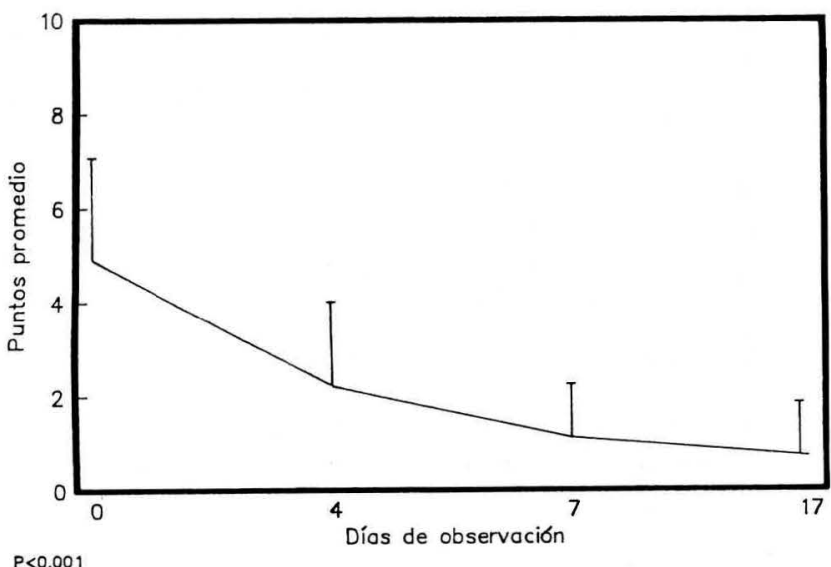

Evaluación global de la severidad de los síntomas

En la Tabla 3, Figura 2 se observa la distribución porcentual de pacientes según la severidad de los síntomas en donde al final, día 17 , en el $61 \%$ de los casos $(\mathrm{n}=11)$ desaparecieron los síntomas y en un $33.3 \%(\mathrm{n}=6)$, fueron de carácter leve. Sólo un caso permaneció con una sintomatología moderada. La disminución de los síntomas fue estadísticamente significativa $(\mathrm{p}<0.001)$.

TABLA 3

EVALUACION GLOBAL DE SEVERIDAD DE LOS SINTOMAS

\begin{tabular}{|rrrrrrrrrrr|}
\hline \multicolumn{4}{c}{ Ausente } & \multicolumn{2}{c|}{ Leve } & \multicolumn{2}{c|}{ Moderada } & \multicolumn{2}{c|}{ Severa } \\
Días & N & $\%$ & N & $\%$ & N & $\%$ & N & $\%$ & X & SD \\
\hline 0 & 0 & 0.0 & 4 & 22.2 & 8 & 44.4 & 6 & 33.3 & 4.9 & 2.2 \\
4 & 6 & 33.3 & 11 & 61.1 & 1 & 5.6 & 0 & 0.0 & 2.2 & 2.0 \\
7 & 6 & 33.3 & 11 & 61.1 & 1 & 5.6 & 0 & 0.0 & 1.1 & 1.1 \\
17 & 11 & 61.1 & 6 & 33.3 & 1 & 5.6 & 0 & 0.0 & 0.7 & 1.0 \\
\hline
\end{tabular}

\section{Evaluación global de síntomas} por puntos promedio

Al comenzar el estudio los pacientes tenían un promedio de $4.9+/-2.2$ puntos y a través del estudio disminuyeron en el promedio a $0.7+/-1$ puntos, siendo estadísticamente significativo el descenso del total de puntos promedios del grupo de pacientes $(p<0.001)$ (Tabla 3, Figura 3).
FIGURA 2

EVALUACION GLOBAL DE LA TERAPIA



FIGURA 3

FRECUENCIA DE GERMENES

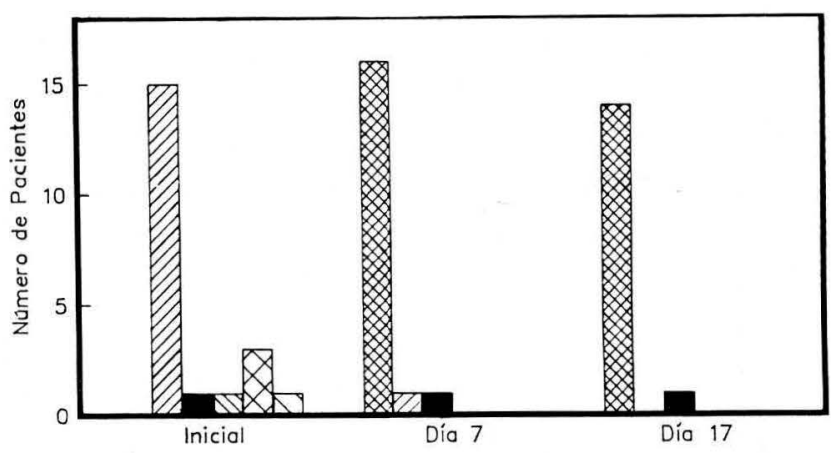

Qusente $\square$ E.coli Proteus Mirabilis $\mathbb{S}$ S. epidermidis $\square$ Citrobocter diversus $\square$ Citrobacter freundii $P<0.001$

Frecuencia de gérmenes

Todos los urocultivos fueron positivos para un germen al inicio del estudio, siendo el más frecuente E. coli en el $83.3 \%(n=15)$. En el día 7 dos casos presentaban cultivo positivo, uno para E. coli, el cual desapareció en el tercer control y otro para Proteus mirabilis, el cual persistió en el control del día 17 aun cuando el antibiograma reportaba al germen sensible a la gentamicina (Tabla 4, Figura 4).

En el control del día 4, sintomatología global estuvo entre leve y moderada para el $94 \%$ de las pacientes, en el control del día 7 el $88.9^{\circ}$ de los urocultivos estaban negativos y la sintomatología 
global entre ausente y moderada en el $100 \%$ de los casos y en el día 17 estuvieron negativos en el $94.4 \%$ de los urocultivos y la sintomatología global estuvo ausente en el $61 \%$ de las pacientes y entre ausente y leve en el $95 \%$ siendo estadísticamente significativa la correlación entre la negatividad del cultivo y la respuesta clínica $(\mathrm{p}<0.001)$.

TABLA 4

FRECUENCIA DE GERMENES

\begin{tabular}{|lrrrrrrr|}
\hline \multicolumn{1}{|c}{ Días } & \multicolumn{1}{c}{ 0 } & & 7 & & \multicolumn{1}{c|}{17} & \\
& $\mathrm{~N}$ & $\%$ & $\mathrm{~N}$ & $\%$ & $\mathrm{~N}$ & $\%$ \\
\hline Negativo & 0 & 0.0 & 16 & 88.9 & 17 & 94.4 \\
E. coli & 15 & 83.3 & 1 & 5.6 & 0 & 0.0 \\
Proteus mirabilis & 1 & 5.6 & 1 & 5.6 & 1 & 5.6 \\
Staphilococo epidermidis & 1 & 5.6 & 0 & 0.0 & 0 & 0.0 \\
Citrobacter diversus & 3 & 16.7 & 0 & 0.0 & 0 & 0.0 \\
Citrobacter freundii & 1 & 5.6 & 0 & 0.0 & 0 & 0.0 \\
\hline
\end{tabular}

FIGURA 4

EVALUACION DE SINTOMAS

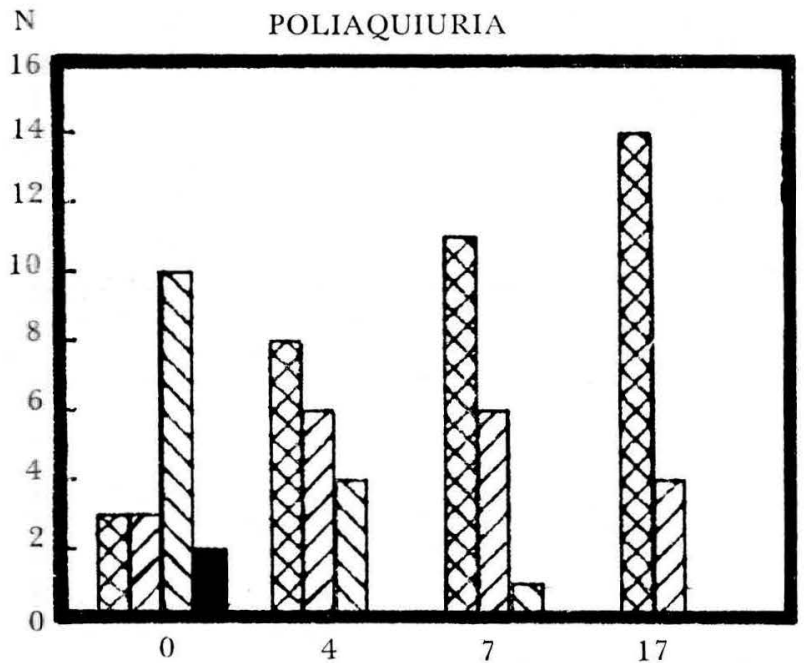

N DISURIA



0

4

7
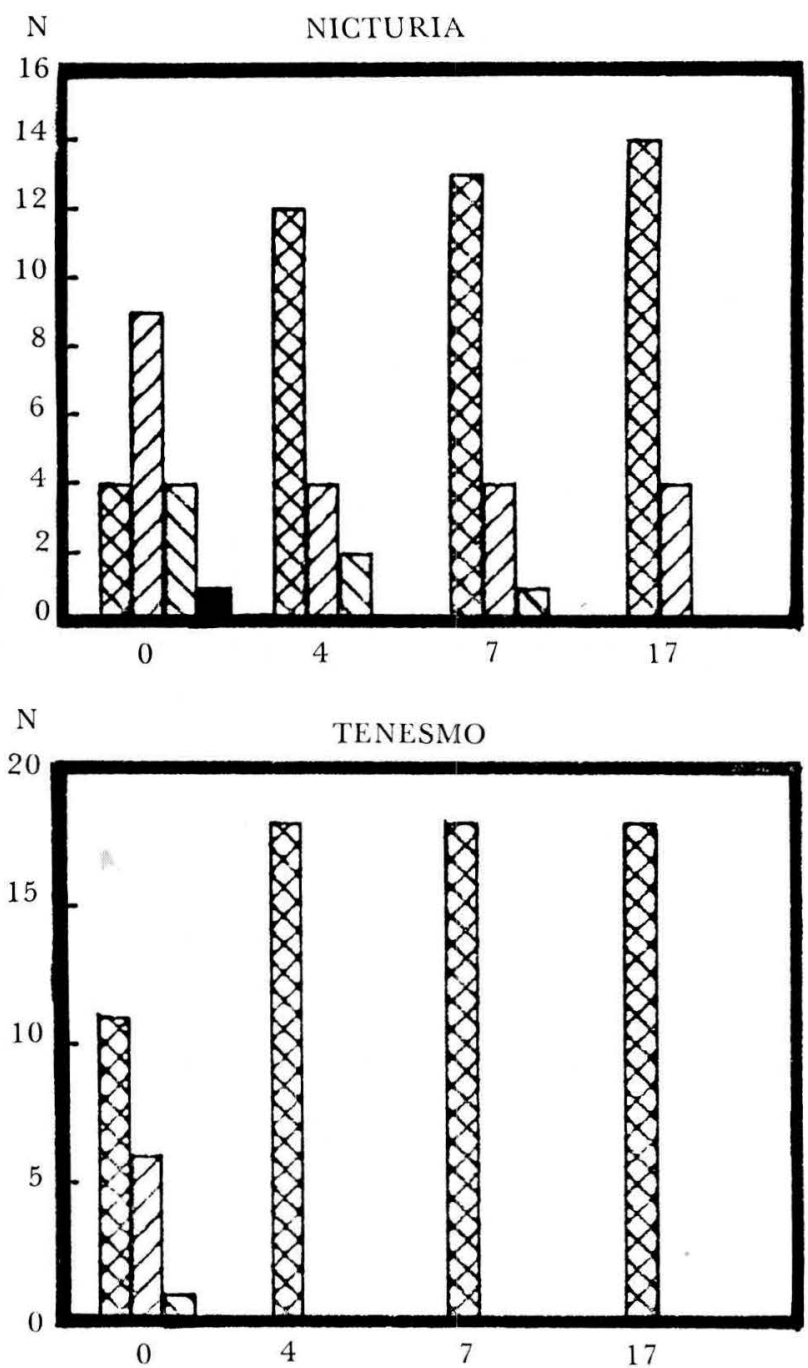

Días de observación
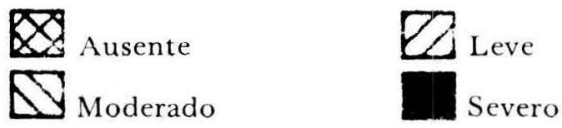

$\mathrm{P}<0.001$

La tolerancia fue excelente $(100 \%)$, no se presentaron efectos secundarios.

Evaluación global de la terapia

De las 18 pacientes evaluables el $88.9 \%$ $(\mathrm{n}=16)$, respondieron satisfactoriamente (excelente y bueno), dos pacientes tuvieron una respuesta regular, una de ellas con cultivo positivo persistente (Proteus mirabilis), con disminución de síntomas en un $50 \%$ y, la otra, con negativización del urocultivo pero pobre respuesta clínica. Los resultados fueron estadísticamente significativos $(\mathrm{p}<0.001)$ (Figura 5). 
FIGURA 5

\section{EVALUACION GLOBAL DE SEVERIDAD DE LOS SINTOMAS}

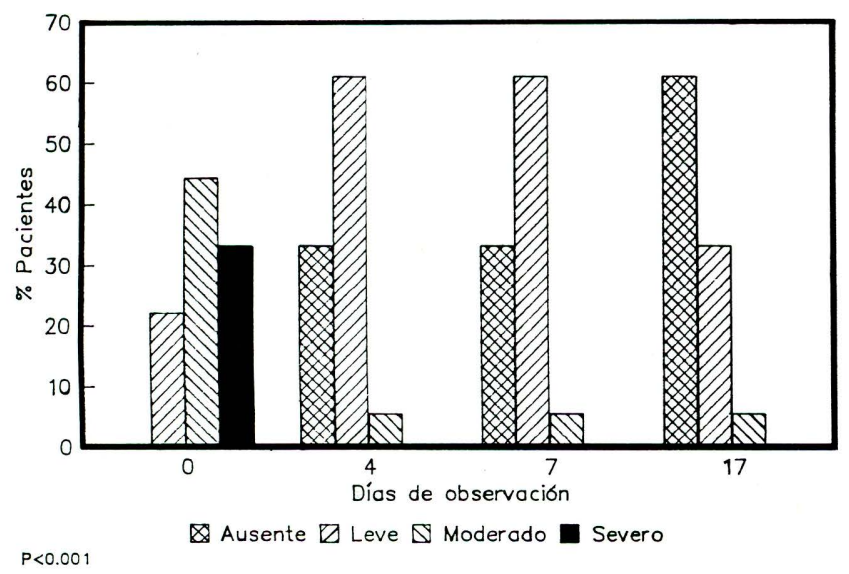

\section{DISCUSION}

Los resultados del presente estudio muestran que se cumplieron los objetivos propuestos, y el esquema terapéutico en investigación adecuado para el tratamiento de las infecciones del tracto urinario con los gérmenes causales frecuentes.

Los gérmenes aislados en los urocultivos del estudio se correlacionan con los encontrados por otros autores en estudios similares, siendo en todos los más frecuentes E. coli, los Proteus y/o Klebsiella o el Citrobacter $(7,8)$. La correlación entre la negatividad del urocultivo y la mejoría clínica es evidente al día 17 , luego para usos prácticos, el uro- cultivo intermedio no es realmente necesario, pero sí se justifica la realización de éste en el último control, ya que verifica al esterilización de la orina.

La persistencia del Proteus mirabilis en una paciente, en el último urocultivo, se puede atribuir a una patología asociada no detectada o a bajas concentraciones del antibiótico para este germen, lo cual llevaría a que se tomara como alternativa el asociar otro antibiótico al esquema terapéutico, en este caso. En la paciente en que no se presentó una satisfactoria evolución clínica, con urocultivo negativo permite pensar que la infección urinaria puede ser coincidente a otra patología de base en estudio.

Al comparar proporcionalmente la respuesta terapéutica del presente estudio $(88.9 \%)$, es similar con la reportada en otros trabajos, en los que utilizaron monodosis de gentamicina, $93 \%(9), 85 \%(1)$, y $82 \%(2)$.

En resumen, el uso de monodosis de gentamicina a $2 \mathrm{mg} / \mathrm{kg}$ de peso/día durante 7 días es aconsejable para el tratamiento de las infecciones del tracto urinario.

La gentamicina continúa siendo un antibiótico de primera elección en el tratamiento de este tipo de pacientes y patologías, sin presentar efectos secundarios y cubriendo el espectro común de gérmenes causales.

Es recomendable tomar un urocultivo con antibiograma al iniciar el tratamiento y una semana después de finalizado, para verificar la esterilidad de la orina.

\section{BIBLIOGRAFIA}

1. ANGELOV, A. ; BARRIENTOS, G. y cols. Dosis única diaria 1 de Refobacin en el tratamiento de infecciones del tracto urinario. Rev. Med. Semanal Munich 1980; 6212-214.

2. PRAT, V.; BOHUSLAV, V. ; HATALA, M. Treatment with a single daily dose of gentamicin in urinary tract infection in relation to the site of infection. Infection 1978; 6 (1): 29-31.

3. HATAL, M. ; MORAVEK, J. y cols. Terapia de dosis única diaria de gentamicina en pielonefritis experimental. Infection 1977; 5 (4): pp.

4. BACK, J. ; CALENSNICK, B. y cols. Pharmacology of gentamicin, a new broad-spectrum antibiotic. AntimicrAg Chemother 1963: 138-147.

5. FERREIRA, A. y cols. Gentamicin in urinary tract infections. Proc 8th Int Congr Chemother Athens 1973; 3: 482-489.
6. LABOVITZ, E. y cols. Single doses daily gentamicin therapy in urinary tract infections J infect Dis 1976; 134: 142-145.

7. ZELLNER, P. R. ; METZGER, E. Optierte gentamicyn-monotherapie bei brandverletzen durch bestimmung der serumkonzentration. Symposium in Gentamycin Dusseldorf Febrero 1976 .

8. KLASTERSKY, J. ; CAPPEL, R. Efecto de la gentamicina en el tratamiento de infecciones severas por gram negativos. Schweiz. Rundschau Med (Praxis) 1973; 62: 537-542.

9. SHARMA, S. D. Gentamicina inyectable: Dosis única diaria en infecciones del tracto urinario. Current Ther Res 1984; 35. 